\title{
Weaning off mechanical ventilation: much less an art, but not yet a science
}

\author{
Paolo Navalesi ${ }^{1,2}$, Andrea Bruni ${ }^{1,2}$, Eugenio Garofalo ${ }^{1,2}$, Eugenio Biamonte ${ }^{2}$, Federico Longhini ${ }^{1,2}$, \\ Pamela Frigerio $^{3}$
}

\begin{abstract}
${ }^{1}$ Anesthesia and Intensive Care, Department of Medical and Surgical Sciences, Magna Graecia University, Catanzaro, Italy; ${ }^{2}$ Unit of Anesthesia and Intensive Care, University Hospital Mater Domini, Catanzaro, Italy; ${ }^{3}$ ASST Grande Ospedale Metropolitano Niguarda, Milano, Italy

Correspondence to: Paolo Navalesi, MD, FERS. Anesthesia and Intensive Care, Department of Medical and Surgical Sciences, Magna Graecia University, Viale Europa, Loc. Germaneto, Catanzaro 88100, Italy. Email: pnavalesi@gmail.com.

Provenance: This is an invited article commissioned by the Section Editor Dr. Guo-Wei Tu (Department of Critical Care Medicine, Zhongshan Hospital, Fudan University, Shanghai, China).

Comment on: Subirà C, Hernández G, Vázquez A, et al. Effect of Pressure Support vs T-Piece Ventilation Strategies During Spontaneous Breathing Trials on Successful Extubation Among Patients Receiving Mechanical Ventilation: A Randomized Clinical Trial. JAMA 2019;321:2175-82.
\end{abstract}

Submitted Aug 27, 2019. Accepted for publication Sep 12, 2019.

doi: $10.21037 / \mathrm{atm} .2019 .09 .83$

View this article at: http://dx.doi.org/10.21037/atm.2019.09.83

The process of liberating patients from mechanical ventilation, commonly referred to as weaning, consists of different steps (1).

The first step aims to ascertain, based on clinical evaluation and assessment of physiologic variables, patient's readiness to undergo a spontaneous breathing trial (SBT) (1).

When the clinical and physiologic criteria are satisfied, the patient is considered ready for the second step, i.e., SBT execution. The manner in which the SBT is performed varies among studies and, likely, among centers in the daily routine practice, with respect to both trial duration and modality of application. Trial duration generally varies between 30 and 120 minutes (2). Regarding the modality of application, the SBT can be either completely unsupported or partially supported. In the former case, commonly referred to as T-tube trial (2), the patient is disconnected from the ventilator and allowed to breathe through a T-tube circuit, or maintained on the ventilator through the use of flow-by, setting pressure support (PS) and positive endexpiratory pressure (PEEP) at $0 \mathrm{cmH}_{2} \mathrm{O}$. Partially supported SBTs involve the use of low levels of continuous positive airway pressure (CPAP) (3), or low to moderate amounts of inspiratory assistance, generally applied in PS mode $\left(5-8 \mathrm{cmH}_{2} \mathrm{O}\right)$, with $(4,5)$ or without (6) associated PEEP. Less frequently, forms of automatic compensation for the nominal additional resistance caused by the endotracheal tube (ET) are used (7).

Third step, when SBT is successful, also based on clinical evaluation and assessment of physiologic variables, and no further contraindications interfere, the patient is extubated. The time to define whether extubation is successful varies among studies from 24 hours and one week, the most common threshold being 48 or 72 hours (1).

The weaning process has been classified as (I) simple, i.e., the patient passes SBT on the first attempt and is successfully extubated; (II) difficult, i.e., the patient requires up to three SBTs or as long as 7 days after the first SBT attempt to achieve successful extubation; (III) prolonged, i.e., the patient fails at least three SBT or requires more than 7 days after the first SBT attempt (1).

Since dissimilarities of the criteria adopted at each of these stages may remarkably influence the outcome of weaning (step 1 and 2) and extubation (step 2 and 3), it is worth considering a few issues.

With respect to step 1, the criteria for readiness influence the time point at which SBT is attempted. First, the greater the number of variables considered, the lower the likelihood of a positive assessment. Second, accepting more liberal criteria (for instance, a maximum respiratory rate of 30 breaths/min rather than 24 breaths/min) increases the probability of considering a patient ready for SBT. It should also be considered that adopting fixed criteria regardless of 


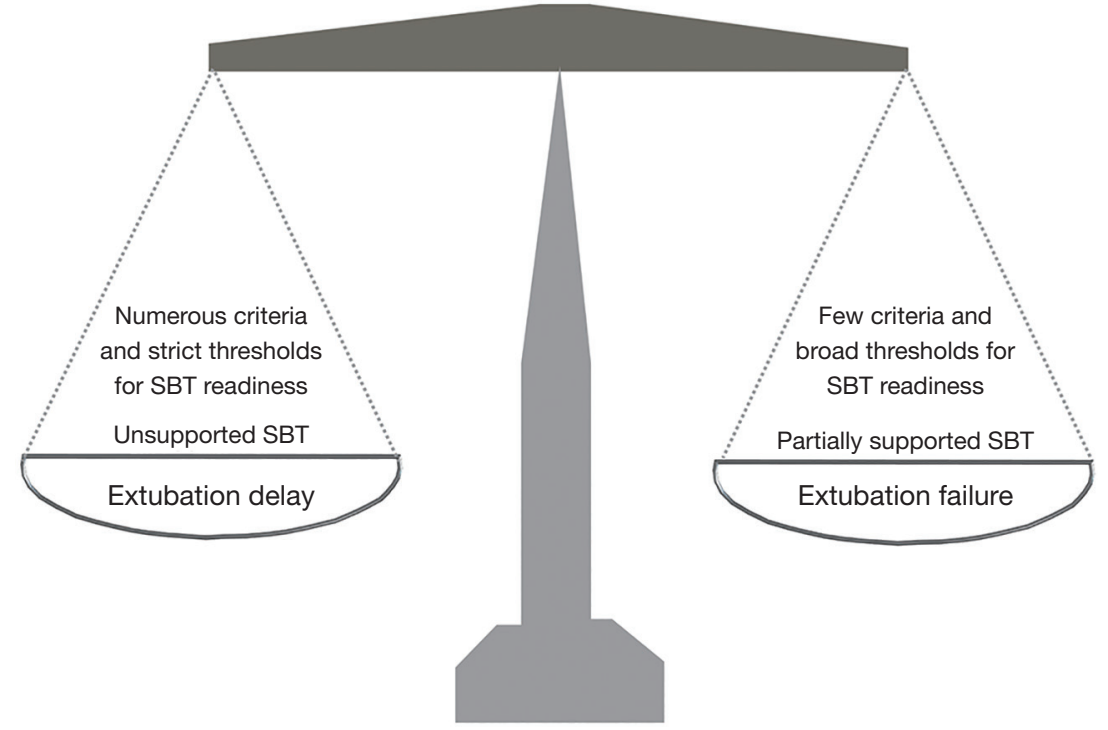

Figure 1 Factors affecting the balance between risk of unduly delaying extubation and risk of extubation failure.

the underlying disorder may generate discrepancies. For example, for a required oxygen saturation $\left(\mathrm{SaO}_{2}\right) \geq 92 \%$ with an inspiratory oxygen fraction $\left(\mathrm{FiO}_{2}\right) \leq 0.4$, the chances of satisfying this criterion for patients with an underlying chronic respiratory failure are fewer than for patients not suffering of chronic respiratory disorders. If on the one hand fewer and more liberal criteria should intuitively increase the likelihood of earlier SBT attempt, leading, potentially, to anticipated extubation and reduced length of mechanical ventilation, on the other hand one might expect a greater rate of SBT success when more numerous variables considered and stricter criteria are adopted, as described in Figure 1.

Let's jump to step 3. Extubation failure may, on the one hand, be consequent to the incapacity to maintain spontaneous unassisted breathing after removal of the endotracheal tube, which implies the SBT failed to detect patient's incapacity to breathe on her/his own after extubation (false positive), predominantly consequent to an increase of the load imposed on the respiratory muscles, cardiac failure or glottic edema secondary to extubation. If immediate endotracheal intubation is not strictly indicated, a limited number of these patients may respond to noninvasive ventilation (NIV) (8). Indeed, these patients need (noninvasive) mechanical ventilation without necessarily requiring an artificial airway. When the risk of developing post-extubation is considered to be high, early prophylactic NIV has the potential to facilitate extubation success (8). High flow oxygen therapy (HFOT) through nasal cannula has been also shown effective in improving extubation outcome (9). On the other hand, extubation failure can be consequent to the inability to maintain the upper airway patent, or to failure to effectively clear secretions. Both these conditions are not detectable by SBT and require additional evaluations to be predicted. Indeed, these patients need an artificial airway without necessarily requiring mechanical ventilation.

Now, let's move backward to step 2. Ideally, a diagnostic test should detect a given condition with $100 \%$ sensitivity and $100 \%$ specificity. Although SBT is a diagnostic test, its specificity cannot be ascertained, as it would be clearly unethical extubating patients failing the test (10). Anyhow, SBT should be strict enough to prevent false positives (patients failing extubation despite passing SBT), while not too conservative to avert the risk of erroneously considering the patient unable to breath spontaneously and unduly prolonging mechanical ventilation. In fact, both SBT failure and reintubation consequent to development postextubation respiratory failure constitute major clinical problem affecting patients' outcome and increasing the economic burdens. Failure to discontinue mechanical ventilation is associated with increased morbidity and mortality, in particular for patients experiencing prolonged weaning (11). Extubation failure, reported to occur between $15 \%$ and $18 \%$ of planned extubation, significantly increases the risk of death (12), irrespective of underlying disease and 
clinical conditions at the time of extubation (13).

With respect to trial duration, two studies did not detect significant difference between trials of 30 and 120 minutes with both unsupported (2) and partially supported (6) SBT. Worth mentioning, Esteban et al. found that, irrespective of trial duration, failure occurred within the first 20 minutes in the course of SBT (2).

Much more controversy exists regarding the modality of trial application. To avert the risk of SBT failure and limit the possibility of unduly prolong mechanical ventilation, some clinicians prefer partially supported SBTs. The application of PEEP/CPAP is founded on the questionable aim of counterbalancing the loss of physiologic PEEP from intermittent vocal cords narrowing (10). In addition, in patients with dynamic hyperinflation, such as chronic obstructive pulmonary disease (COPD) patients, low PEEP/CPAP levels may promote SBT success by offsetting to some extent the threshold load caused by intrinsic PEEP secondary to dynamic hyperinflation (14). The inspiratory assistance aims to offset the additional resistive load caused by the ET (15), which varies, nonetheless, depending on ET size (16) and presence of concrete secretions within the ET lumen (17). However, after extubation the upper airways are naturally inflamed, leading to an increased resistance than expected; indeed, the work of breathing exerted during an unsupported SBT was shown to be closer to that observed after extubation with unsupported SBT, as opposed to partially supported SBT $(18,19)$.

These data rise up concern that some patients who tolerate a partially supported SBT may be unable to maintain unsupported spontaneous breathing after extubation, thereby increasing the risk of post-extubation respiratory and need for reintubation, potentially associated with life-threatening complications (10). Actually, a recent physiologic meta-analysis, including 16 studies that measured work of breathing and inspiratory efforts in 239 patients, confirmed that during unsupported SBT the workload imposed on the respiratory muscles is greater than with partially supported SBT. However, work of breathing and inspiratory effort with the former resulted to more accurately reflect the physiologic conditions after extubation than with the latter (20). These results, though limited by the relatively small number of patients and by the high heterogeneity, challenge the notion that an unsupported SBT is a "stress" with a higher potential of failure.

Contemporarily, however, another meta-analysis of 31 studies, overall including 3,541 patients, has been published, which compared unsupported and partially supported SBTs with respect to SBT and extubation outcomes, and reintubation rate (7). While there were no differences in SBT outcome and rate of reintubation, the patients undertaking partially supported SBTs showed a significant $5 \%$ greater rate of successful extubation, as opposed to those undergoing unsupported STBs (7). In theory, we might consider a partially supported SBT to reduce the probability of delaying extubation and increase the risk of extubation failure, while, contrariwise, an unsupported SBT to augment and lessen the chance of extubation delay and failure, respectively (Figure 1).

These notions have been recently challenged by Subirà et al. who have published in JAMA the largest randomized controlled trial ever done to compare different SBTs. The authors hypothesize that a less demanding partially supported SBT, i.e., PS $8 \mathrm{cmH}_{2} \mathrm{O}$ for 30 minutes, would be superior to an unsupported SBT lasting 120 minutes (21). The investigators enrolled 1,153 adult patients receiving invasive mechanical ventilation for more than 24 hours and meeting predefined criteria for undergoing first SBT attempt. The primary outcome was successful extubation, i.e., no need for invasive mechanical ventilation 72 hours after the first SBT. Secondary outcomes were rate of reintubation among patients extubated after the first SBT, intensive care unit (ICU) and hospital lengths of stay, and hospital and 90-day mortality, while ICU mortality was a post hoc outcome (21).

The low demanding strategy led to a significantly higher rate of SBT success $(92.5 \%$ vs. $82.3 \%$, and successful extubation (82.3\% vs. 74\%, 95\% CI: 3.4-13\%; $\mathrm{P}<0.001)$, The Kaplan-Meier curves, built on a 72-hour time scale, also show a significantly greater rate of successful extubation in the partially supported SBT group (hazard ratio, 1.54; 95\% CI: $1.19-1.97 ; \mathrm{P}<0.001)$. Reintubation within 72 hours, and ICU and hospital length of stay were similar between the two groups. Hospital and 90-day mortalities were lower in the partially supported SBT group, while ICU mortality was no different (21). Read in a different way, compared to unsupported SBT, partially supported SBT increases the likelihood for a patient to get into the category of simple weaning. These results, coming from a large multicenter RCT, are clearly important, but not definitive, as also neatly outlined in an accompanying editorial by Girard and Burns (22), for the following reasons.

First, as above mentioned, SBT outcome is influenced by number, type and thresholds of the criteria adopted for assessing readiness for SBT. An observational multicenter study including 2,714 intubated patients satisfying criteria 
for weaning readiness, found that $45 \%$ of patients failed at least one attempt, encompassing conditions of difficult (39\%) and prolonged (6\%) weaning (11). This is not a criticism for the readiness criteria chosen by the authors, nor a specific limit of this study only. Nevertheless, we should bear in mind that this represents a limitation for the generalizability of these findings.

Second, the study considers only the first SBT attempt. In fact, "additional SBTs were not protocolized, and mode and duration were left to the discretion of attending teams" and similarly there were no specific indications for the patients who fail extubation. Therefore, these results cannot be extrapolated to patients experiencing more problematic weaning, i.e., difficult or prolonged.

Third, prophylactic use of NIV and HFOT have been shown to improve the outcome of extubation in patients at high (8) and low risk of extubation failure, respectively (23). Noteworthy, in this trial the use of prophylactic NIV or HFOT after extubation was left to the attending physicians' choice before randomization and resulted to be more commonly used after extubation in the low demanding SBT group, as opposed to unsupported SBT (25\% vs. $19 \% ; \mathrm{P}=0.01)(22)$, which offers two intriguing cues: (I) the physicians were more concerned of the outcome of extubation in the former group than in the latter; (II) routine use of these two techniques may substantially affect extubation outcome limiting the risk of developing postextubation respiratory failure (prophylactic use)in selected high risk patients, and, in a limited subgroup of patients experiencing post-extubation respiratory failure, averting the need for reintubation. Since the same working group involved in the present study showed in a previous RCT that HFOT, compared to standard oxygen, significantly reduces rates of post-extubation respiratory failure and reintubation, we wonder how extensive HFOT application could affect the results of the present trial.

Fourth, based on the available information, the observed differences in hospital and 90-days mortalities between groups cannot be explained. In fact, the rate of reintubation, the strongest determinant of adverse outcome, is not different between the two groups, while data about overall duration of invasive mechanical ventilation, the second determinant of adverse outcome, are not provided by the authors, either in the main manuscript or in the online supplements. Nonetheless, the similar lengths of ICU and Hospital stay seem to suggest that the difference were likely not significant, if existing.

Judicious use of evidence should never exclude individualized behaviors for specific patients. RCTs are the unavoidable foundation for clinical evidence and the groundwork of population-based medicine. However, applying the average effects, measured as population means, to individual patients or subgroups departing from the population average does not necessarily lead to the right treatment for the right patient, masking the heterogeneity of effects, i.e., clear benefits for some, little benefits for many, and harm for a few (24). This holds particularly true when dealing with conditions that are not strictly related to a specific disease, as it is the case for weaning.

In 1986, Prof Joseph Milic-Emili wrote a famous editorial well summarized by the title "Is weaning an art or a science?" (25). After more than three decades and many studies, we believe weaning today is much less an art, but not yet a science.

\section{Acknowledgments}

None.

\section{Footnote}

Conflicts of Interest: The authors have no conflicts of interest to declare.

Ethical Statement: The authors are accountable for all aspects of the work in ensuring that questions related to the accuracy or integrity of any part of the work are appropriately investigated and resolved.

\section{References}

1. Boles JM, Bion J, Connors A, et al. Weaning from mechanical ventilation. Eur Respir J 2007;29:1033-56.

2. Esteban A, Alia I, Tobin MJ, et al. Effect of spontaneous breathing trial duration on outcome of attempts to discontinue mechanical ventilation. Spanish Lung Failure Collaborative Group. Am J Respir Crit Care Med 1999;159:512-8.

3. Longhini F, Maugeri J, Andreoni C, et al. Electrical impedance tomography during spontaneous breathing trials and after extubation in critically ill patients at high risk for extubation failure: a multicenter observational study. Ann Intensive Care 2019;9:88.

4. Vaschetto R, Longhini F, Persona P, et al. Early extubation followed by immediate noninvasive ventilation vs. standard extubation in hypoxemic patients: a randomized clinical 
trial. Intensive Care Med 2019;45:62-71.

5. Navalesi P, Frigerio P, Moretti MP, et al. Rate of reintubation in mechanically ventilated neurosurgical and neurologic patients: evaluation of a systematic approach to weaning and extubation. Crit Care Med 2008;36:2986-92.

6. Perren A, Domenighetti G, Mauri S, et al. Protocoldirected weaning from mechanical ventilation: clinical outcome in patients randomized for a $30-\mathrm{min}$ or $120-\mathrm{min}$ trial with pressure support ventilation. Intensive Care Med 2002;28:1058-63.

7. Burns KEA, Soliman I, Adhikari NKJ, et al. Trials directly comparing alternative spontaneous breathing trial techniques: a systematic review and meta-analysis. Crit Care 2017;21:127.

8. Rochwerg B, Brochard L, Elliott MW, et al. Official ERS/ ATS clinical practice guidelines: noninvasive ventilation for acute respiratory failure. Eur Respir J 2017. doi: 10.1183/13993003.02426-2016.

9. Renda T, Corrado A, Iskandar G, et al. High-flow nasal oxygen therapy in intensive care and anaesthesia. Br J Anaesth 2018;120:18-27.

10. Tobin MJ. Extubation and the myth of "minimal ventilator settings". Am J Respir Crit Care Med 2012;185:349-50.

11. Peñuelas O, Frutos-Vivar F, Fernandez C, et al. Characteristics and outcomes of ventilated patients according to time to liberation from mechanical ventilation. Am J Respir Crit Care Med 2011;184:430-7.

12. Epstein SK, Ciubotaru RL. Independent effects of etiology of failure and time to reintubation on outcome for patients failing extubation. Am J Respir Crit Care Med 1998;158:489-93.

13. Thille AW, Harrois A, Schortgen F, et al. Outcomes of extubation failure in medical intensive care unit patients. Crit Care Med 2011;39:2612-8.

14. Reissmann HK, Ranieri VM, Goldberg P, et al. Continuous positive airway pressure facilitates spontaneous breathing in weaning chronic obstructive pulmonary disease patients by improving breathing pattern and gas exchange. Intensive Care Med 2000;26:1764-72.

Cite this article as: Navalesi P, Bruni A, Garofalo E, Biamonte E, Longhini F, Frigerio P. Weaning off mechanical ventilation: much less an art, but not yet a science. Ann Transl Med 2019;7(Suppl 8):S353. doi: 10.21037/atm.2019.09.83
15. Brochard L, Rua F, Lorino H, et al. Inspiratory pressure support compensates for the additional work of breathing caused by the endotracheal tube. Anesthesiology 1991;75:739-45.

16. Navalesi P, Hernandez P, Laporta D, et al. Influence of site of tracheal pressure measurement on in situ estimation of endotracheal tube resistance. J Appl Physiol (1985) 1994;77:2899-906.

17. Wright PE, Marini JJ, Bernard GR. In vitro versus in vivo comparison of endotracheal tube airflow resistance. Am Rev Respir Dis 1989;140:10-6.

18. Straus C, Louis B, Isabey D, et al. Contribution of the endotracheal tube and the upper airway to breathing workload. Am J Respir Crit Care Med 1998;157:23-30.

19. Mahul M, Jung B, Galia F, et al. Spontaneous breathing trial and post-extubation work of breathing in morbidly obese critically ill patients. Crit Care 2016;20:346.

20. Sklar MC, Burns K, Rittayamai N, et al. Effort to Breathe with Various Spontaneous Breathing Trial Techniques. A Physiologic Meta-analysis. Am J Respir Crit Care Med 2017;195:1477-85.

21. Subirà C, Hernandez G, Vazquez A, et al. Effect of Pressure Support vs T-Piece Ventilation Strategies During Spontaneous Breathing Trials on Successful Extubation Among Patients Receiving Mechanical Ventilation: A Randomized Clinical Trial. JAMA 2019;321:2175-82.

22. Girard TD, Burns KEA. Revisiting, Reframing, and Casting a New Light on Liberation From Mechanical Ventilation. JAMA 2019;321:2167-9.

23. Hernández G, Vaquero C, Gonzalez P, et al. Effect of Postextubation High-Flow Nasal Cannula vs Conventional Oxygen Therapy on Reintubation in Low-Risk Patients: A Randomized Clinical Trial. JAMA 2016;315:1354-61.

24. Kravitz RL, Duan N, Braslow J. Evidence-based medicine, heterogeneity of treatment effects, and the trouble with averages. Milbank Q 2004;82:661-87.

25. Milic-Emili J. Is weaning an art or a science? Am Rev Respir Dis 1986;134:1107-8. 